\title{
Suitability of EVAR with Hostile Neck
}

\author{
HOSSAM Z. YOUSIF, M.D.; AHMED M. EL-MAHROUKY, M.D. and MOHAMMED ALI HASSAN, M.D. \\ The Department of Vascular Surgery, Faculty of Medicine, Cairo University
}

\begin{abstract}
Background: Endovascular treatment of abdominal aortic aneurysms (EVAR) is now considered first line of treatment for abdominal aortic aneurysm (AAA) mainly due to the lower morbidity and mortality, hostile neck is a major obstacle in treating such patients, however many endovascular methods are available to overcome such obstacle, EVAR out of IFU is considered one of the lines of management. Further evaluation is needed.
\end{abstract}

Aim of Study: Aim of this study was to evaluate the effectiveness of endovascular treatment of AAA with hostile neck anatomy, with commenting on the perioperative complications, mortality and morbidity.

Material and Methods: This study was done retrospectively over a period of 18 months including 10 patients who had endovascular treatment of abdominal aortic aneurysms with hostile neck anatomy.

Results: 10 patients were included in this study primary technical success was achieved in $90 \%$ of cases while the last case needed aortic cuff as an adjunctive procedure. One case required re-intervention within 30 days post-operative due to limb graft occlusion, managed by femoro-femoral bypass. At one year follow up mortality was $10 \%$.

Conclusion: EVAR for AAA patients with HNA is feasible; patients with single feature of HNA showed the best outcome, while patients with more than one feature required the usage of adjunctive techniques for proximal sealing and were more liable to develop post-operative complications.

Key Words: AAA - Hostile neck - Endovascular treatment.

\section{Introduction}

ENDOVASCULAR treatment of abdominal aortic aneurysms (EVAR) is now considered first line of treatment for abdominal aortic aneurysm (AAA) mainly due to the lower morbidity and mortality associated with this procedure compared to standard open repair. Studies have shown that $70 \%$ of cases of AAA can be managed by EVAR using currently available stent graft [1]

Correspondence to: Dr. Hossam Z. Yousif, The Department of Vascular Surgery, Faculty of Medicine, Cairo University
However hostile neck is still an important barrier to offer EVAR to all cases of AAA as specific parameters in the neck as length, diameter and angulation, thrombus burden, calcification are prerequisites for successful and safe sealing as recommended by manufactures of stent grafts in the instructions for use (IFU) $[2,3]$.

To overcome the problem of hostile neck and to be able to manage cases with hostile neck by endovascular techniques, several modifications have been tried including fenestrated EVAR and chimney techniques require high endovascular skills and tertiary centers in addition fenestrated endografts are custom made making them expensive and take several weeks to be available making them not a good option for urgent cases [4-7]

The aim: Of this study was to evaluate the effectiveness of endovascular treatment of AAA with hostile neck anatomy (HNA), with commenting on the perioperative complications, mortality and morbidity.

\section{Material and Methods}

\section{Patient selection:}

This study included 10 patients presented to the vascular surgery department at Cairo University teaching hospitals having infra-renal AAA with hostile neck anatomy, between May 2016 and October 2017.

We identified our patients retrospectively through a clinical database collected prospectively. And the opinion of the ethics committee was taken.

Our inclusion criteria included those patients with infra renal AAA with one or more of the characters of hostile neck anatomy. However patients with infra renal AAA with favorable neck anatomy (FNA). Patients who developed an anastomotic pseudoaneurysms post-surgical repair and 
the patients with isolated iliac aneurysms were excluded from our study. All patients underwent C.T.A preoperatively and the neck characteristics were specified to classify the patient either with favorable or unfavorable anatomy according to the instructions of the manufacturers.

\section{Methods and technique:}

The database contains demographic data, medical history, anesthesia, type of access surgical/ percutaneous, type of stent-graft used, technical success, intra- or post-procedural complications, intra- or post-operative mortality, additional intraoperative procedures (surgical or endovascular) and follow-up.

Informed consent was obtained from all patients included in the study.

We used a center-luminal vessel analysis-type reconstruction system to get a better results of the measurements obtained.

EVAR is done through bilateral femoral cutdown then a pigtail catheter is inserted from the contra-lateral side to be used for angiography and kept slightly higher than the level of the renal arteries (L1-L2). Insertion of stiff wire through femoral access followed by insertion of the main module, then adjustment of the main module by adjusting its markers to the lowest renal artery, deployment is continued till the marker of the gate appears. After cannulating the gate with floppy 0.035 " wire, confirmation of the position inside the graft is ensured by progressing the pigtail through the gate and spinning its curved tip.

Afterwards, insertion of the contra-lateral iliac limb and deployment with ensuring an overlap with the main module at least 1.5 stent-cells (iliac extensions are used if needed). Finally, a molding compliant balloon is used to ensure apposition of the graft only at the sealing zones and sites of overlap, and never outside the graft.

We follow-up our patients with CTA at one month and US at six month and CTA at twelve months and then alternately US and CTA every 12 month. We have reviewed all the CTA follow-up of the patients to determine the presence of any complications as in endoleaks, limb thrombosis or graft migration.

\section{The parameters analyzed are:}

- Technical success which is defined as proper positioning of the stent graft and not covering any of the renal arteries and absence of any intraoperative complications as in endoleak Type I.
- Intra and post procedural mortality

- Endoleaks and other complications during the first year after the procedure.

According to the pre-operative CTA we defined hostile Neck as the presence of either short neck with a distance of less than $15 \mathrm{~mm}$ between the lowest renal artery and the beginning of the aneurysm or proximal neck diameter more than $28 \mathrm{~mm}$ or reverse taper which means a gradual neck dilatation of more than two $\mathrm{mm}$ in the first one centimeter after the lowest renal artery and angulation of the neck more than 60 degrees within the first $30 \mathrm{~mm}$ after the lowest renal artery or thrombosis in the neck of more than 50 percent and significant calcification of more than 50 percent of the circumference of the neck.

\section{Results}

The age of the studied patients ranged from 48 to 75 years with a mean of 63.6 years. All the patients were males. Eight patients $(80 \%)$ were found to be smokers while all of them were hypertensive. None of the patients had diabetes. Coronary artery disease (CAD) was found in four patients $(40 \%)$. None of the patients had previous history of kidney disease.

Pre-operative CTA assessment showed eight patients (80\%) with neck length $<15 \mathrm{~mm}$ (Fig. 1), three patients $(30 \%)$ with neck diameter $>28 \mathrm{~mm}$ (Fig. 2), two patients (20\%) with neck angulation $>60^{\circ}$ (Fig. 3), while none of the patients had neither significant neck thrombus nor calcification. The maximum AAA diameter ranged from $50 \mathrm{~mm}$ to $152 \mathrm{~mm}$ with a mean value of $67 \mathrm{~mm}$.

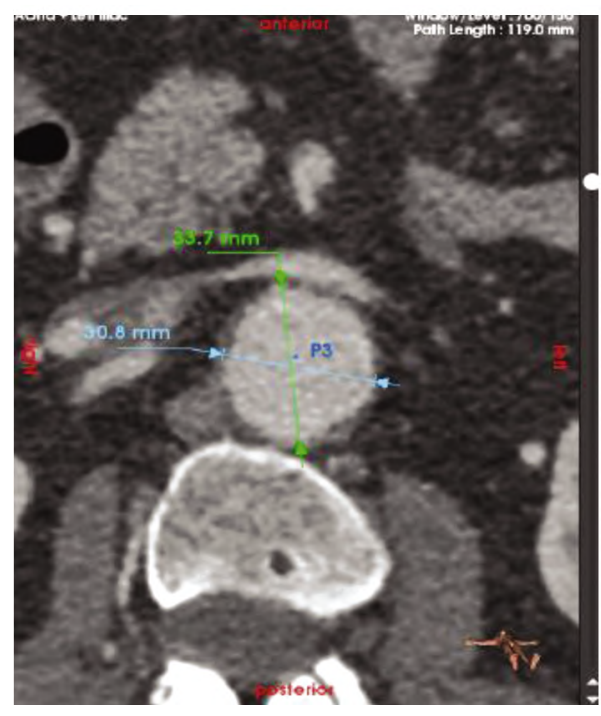

Fig. (1): CTA shows the presence of AAA with "hostile" neck: The neck diameter is $33.7 \mathrm{~mm}$. 


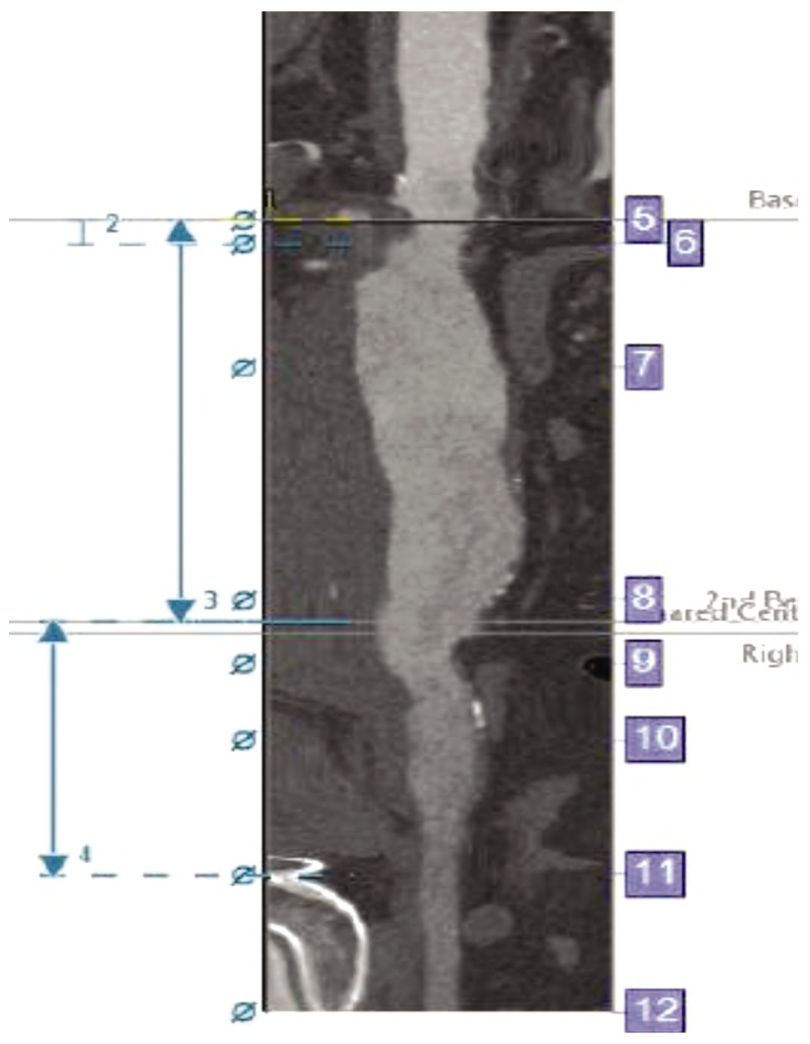

Fig. (2): CTA shows the presence of AAA with "hostile"neck: The neck length is $9 \mathrm{~mm}$.

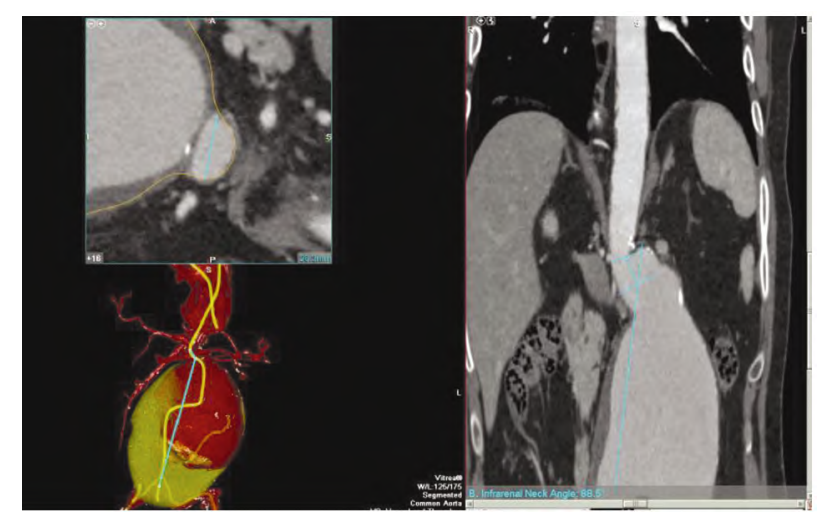

Fig. (3): CTA shows the presence of AAA with "hostile" neck: The angle of the neck between the longitudinal axis of the vessel and the longitudinal axis of the aneurysm is about $88^{\circ}$.

It was found that a single hostile neck feature is present in eight patients (80\%), while one patient had two features (short neck length and angulated neck more than $60^{\circ}$ ) and another patient had three features (short neck length, large proximal neck diameter more than $28^{\circ}$ and angulated neck more than $60^{\circ}$ ).

In all cases general anesthesia was used and the surgical access was the preferred approach.

Six patients $(60 \%)$ were stented by Bolton Treovance device and four patients (40\%) were stented by Medtronic Endurant device and all the patients received a bifurcated endoprosthesis.

Technical success was achieved in nine patients (90\%), while one patient (10\%) achieved assisted technical success by using aortic cuff stent as an adjunctive technique for proximal seal due to the presence of short $(14 \mathrm{~mm})$ and angulated neck $\left(63^{\circ}\right)$.

In the 30 days follow-up period no cases of mortality were noted. One case (10\%) had graft limb occlusion and was successfully treated with femoro-femoral bypass, and no cases developed neither wound infection nor renal insufficiency.

At one year follow-up there was only one type 1 a endoleak and mortality, the patient developed type Ia endoleak at 2 months after treatment due to the caudal migration of the endoprosthesis and was treated by Palmaz stent. Unfortunately it was unsuccessful and the patient died from aneurysm rupture. It is to be noted that this case had three characters of HNA combined short neck $13 \mathrm{~mm}$, angulated neck $88^{\circ}$ and enlarged diameter $32 \mathrm{~mm}$ and the maximum AAA diameter was $152 \mathrm{~mm}$.

\section{Discussion}

AAA with hostile neck anatomy repair is more challenging compared to that with friendly neck anatomy. Unfavorable anatomy increases EVAR complications as graft migration and endoleak Ia [8]. Further insight on how to expand EVAR treatment to more challenging cases with difficult necks and how to prevent potential complications will be the key for improvements in the efficacy and applicability of EVAR [8].

According to our results there is no statistically significant difference regarding demographic data and associated comorbidities in comparison to other studies, Kent et al., reported that dyslipidemia and smoking are the main risk factor in pathogenesis of AAA. They observed a positive association with increasing years of smoking and cigarettes smoked and a negative association with smoking cessation [9]. Hypertension is a predisposing factor for AAA as well as a factor that promotes increase in size and increases rupture risk. Chronic hypertension should be differentiated from hypertension that is present at the time of presentation only [10]

None of our patients had diabetes mellitus which coincides with the literature that although diabetes mellitus has a strong relationship in pathogenesis of atherosclerosis, it has a protective effect against aneurysm formation. Several mechanisms 
have been proposed for the protective effect of diabetes mellitus including hyper-insulinemia and hyperglycemia which can alter the metabolism of the arterial matrix. The effects of pharmacologic therapy used in diabetes management can stabilize mural thrombi and decrease inflammation [11]

Although refinements in endovascular technique have further expanded the indications of EVAR, there are still some limitations. An adequate proximal landing zone is one of the absolute requirements for successful EVAR.

In the current study $10 \%$ of patients had two features of hostile neck anatomy; angulated neck $>60^{\circ}$ and a neck length $<15 \mathrm{~mm}$, this patient had endoleak type I and assisted technical success was achieved by insertion of aortic cuff stent, and $10 \%$ of patients had three features of hostile neck anatomy; angulated neck $>60^{\circ}$, a neck length $<15 \mathrm{~mm}$ and enlarged proximal neck more than $28 \mathrm{~mm}$ this patient had endoleak type 1 and was treated by Palmaz stent. Unfortunately it was unsuccessful and the patient died from aneurysm rupture.

Antoniou et al., reported that the 2 most common reasons for unfeasible EVAR is short neck $(<15 \mathrm{~mm}$ in length) and angulation of more than $60^{\circ}$ especially if both occur together. This is particularly true if both factors are present in the same patient [12]. Matsumoto and his colleagues reported that placement of an endoprosthesis in patients with angulated short neck is technically difficult and they recommend protecting the lowest renal artery by guidewire in it in cases were parallax view may affect accurate placement of the stent graft [13].

It is clear that EVAR is possible in case of isolated hostile neck feature, however the presence of more than one hostile neck factor, requires great care to accurately deploy the stent graft flush with lowest renal to make use of all the length available for fixation and sealing to be successful.

In the current study, one patient (10\%) required the use of adjunctive technique in the form of aortic cuff placement in addition to the trimodular TREVANCE stent (Bolton) due to the presence of short $(14 \mathrm{~mm})$ and angulated neck $\left(63^{\circ}\right)$. Aburahma et al., repoted adjunctive procedures up to $22 \%$ of HNA cases, whereas Matsumoto et al. reported the usage of adjunctive techniques in up to $34 \%$ of HNA cases $[6,13]$.

Patients with hostile neck needed adjunctive procedures to achieve proper sealing.
Successful adjunctive therapies include proximal aortic cuff, high-pressure balloon angioplasty, and balloon-expandable metallic stent placement such as the Palmaz stent (Cordis, Miami Lakes, Fla) [8]

Matsumoto and his colleagues recommend using aortic cuffs instead of Palmaz stents to treat intraoperative type 1a endoleaks as they have noticed that Palmaz stents displace with time and they attributed this to gradual neck dilatation [13]

According to our results technical success was achieved in nine patients $(90 \%)$, while one patient $(10 \%)$ achieved assisted technical success by using aortic cuff stent as an adjunctive technique for proximal seal due to the presence of short $(14 \mathrm{~mm})$ and angulated neck $\left(63^{\circ}\right)$ which coincides with a study done by Cerini et al., that reported technical success in $95.3 \%$ of cases, this may be due to usage of stent grafts with suprarenal fixation which is advantageous in cases with hostile necks [14].

In the follow-up CTA the incidence of endoleak type I was $10 \%$ (one patient), and was treated by Palmaz stent. It is to be noted that the case of type I endoleak occurred in the case where the hostile neck anatomy had three characters combined (short neck $13 \mathrm{~mm}$, angulated neck $88^{\circ}$ and enlarged diameter $32 \mathrm{~mm}$ ).

Matumoto et al., reported $8 \%$ for incidence of endoleak type 1 in HNA cases, whereas Aburahma et al., and Poalo et al., reported incidence of endoleak type 1 in up to $22 \% \& 8.8 \%$ respectively of HNA cases [6.13.14].

In our study cases with intraoperative type Ia endoleak underwent balloon inflation of the proximal neck making sure to keep the balloon in the graft to avoid aortic dissection and was inflated only for few seconds, in our experience this simple maneuver was successful in all cases except one which needed insertion of proximal aortic cuff.

Paolo and his colleagues reported an incidence of $8.8 \%$ for hostile neck patients and they deploy an aorto-uni-iliac device inside and above the EVAR stent graft as management of type Ia endoleak and they recommend this technique as opposed to proximal aortic cuff as they reported 2 cases of failure of aortic cuff to resolve the endoleak Ia and surgical conversion was mandatory in those cases, obviously more studies are needed before widespread use of this technique [14]

Re-intervention within 30 days post operatively was needed in one case due to limb graft occlusion, 
managed by femoro-femoral bypass. Matumoto et al., reported $3 \%$ for incidence of re-intervention in HNA cases, whereas Aburahma et al., reported incidence of re-intervention in up to $28 \%$ of HNA cases $[6,13]$

Limb occlusion of aortic stent-grafts occurs early and is usually due to kinking of the iliac limb, migration or dislocation of an iliac limb or in patients with aortoiliac occlusive disease, a small distal aorta $(<14 \mathrm{~mm})$, or tortuous iliacs. If discovered and managed early, durable outcome is to be expected.

In our study thirty-day post-operative mortality for the ten cases was zero percent. Matumoto et al., reported $1 \%$ for 30 -day mortality, whereas Aburahma et al., reported 3\% 30-day mortality [6,13]

In our study one year mortality was ten percent; a case that had endoleak type Ia and was treated by palmaz stent, postoperatively rupture of the aneurysm resulted in death. Aburahma et al. reported $1 \%$ one year mortality due to aneurysm rupture, whereas Matsumoto et al reported 1\% one year mortality due to aortic dissection $[6,13]$

The mortalities in patients with hostile necks were mainly due to acute myocardial infarction, and were common in patients with intraoperative or postoperative complications.

Although hostile aneurysm neck makes successful deployement of stent graft for AAA more difficult, However analysis of the available data revealed acceptable immediate and early outcomes, in the form of technical success, incidence of type I endoleak, and requirement for reintervention within 30 days of treatment.

Although meta-analyses of late outcome showed inferior results of standard endograft devices in patients with hostile neck anatomy. We conclude that in patients with hostile neck "the high risk of proximal endoleak must be weighed against the risk of treatment modality alternatives".

\section{Conclusion:}

EVAR for AAA patients with HNA is feasible; patients with single feature of HNA showed the best outcome, while patients with more than one feature required the usage of adjunctive techniques for proximal sealing and were more liable to develop post-operative complications so the high risk of proximal endoleak must be weighed against the risk of treatment modality alternatives.

\section{Recommendations}

- HNA of proximal neck in infra-renal AAA should not preclude the option of treatment by EVAR.

- The studied sample needs to be expanded to provide more data for analysis.

- Comparisons of the outcomes of standard EVAR vs fenestrated repair of AAA with hostile neck anatomy may also help surgeons with the optimal management of these patients.

\section{References}

1- LEDERLE F.A., FREISCHLAG J.A., KYRIAKIDES T.C., et al.: Open Versus Endovascular Repair (OVER) Veterans Affairs Cooperative Study Group. Outcomes following endovascular vs open repair of abdominal aortic aneurysm: A randomized trial. JAMA, 3 02: 1535-42, 2009.

2- SICARD G.A., ZWOLAK R.M., SIDAWY A.N., et al.: Society for Vascular Surgery Outcomes Committee. Endovascular abdominal aortic aneurysm repair: Long-term outcome measures in patients at high-risk for open surgery. J. Vasc. Surg., 44: 229-36, 2006.

3- HOBO R., KIEVIT J., LEURS., et al.: EUROSTAR Collaborators. Influence of severe infrarenal aortic neck angulation on complications at the proximal neck following endovascular AAA repair: A EUROSTAR study. J. Endovasc. Ther., 14: 1-11, 2007.

4- MALINA M., RESCH T. and SONESSON B.: EVAR and complex anatomy: An update on fenestrated and branched stent grafts. Scand. J. Surg., 97: 195-204, 2008.

5- ANTONIOU G.A., SCHIRO A., ANTONIOU S.A., et al.: Chimney technique in the endovascular management of complex aortic disease. Vascular., 20: 251-61, 2012.

6- ABU-RAHMA A.F., CAMPBELL J.E., MOUSA A.Y., et al.: Clinical outcomes for hostile versus favorable aortic neck anatomy in endovascular aortic aneurysm repair using modular devices. J. Vasc. Surg., 54: 13-21, 2011.

7- TORSELLO G., TROISI N., DONAS K.P., et al.: Evaluation of the Endurant stent graft under instructions for use vs off-label conditions for endovascular aortic aneurysm repair. J. Vasc. Surg. 54: 300-6, 2011.

8- CHOKE E., MUNNEKE G., MORGAN R., et al.: Outcomes of endovascular abdominal aortic aneurysm repair in patients with hostile neck anatomy. Cardiovascular and Interventional Radiology, 29 (6): 975-980, 2006.

9- KENT K.C., ZWOLAK R.M., EGOROVA N.N., et al.: Analysis of risk factors for abdominal aortic aneurysm in a cohort of more than 3 million individuals. J. Vasc. Surg., 52 (3): 539-548, 2010.

10- VARDULAKI K.A., PREVOST T.C., WALKER N.M., et al.: Incidence among men of asymptomatic abdominal aortic aneurysms: Estimates from 500 screen detected cases. J. Med. Screen., 6 (1): 50-54, 1999.

11- ÅSTRAND H., RYDÉN-AHLGREN Å., SUNDKVIST G., et al.: Reduced aortic wall stress in diabetes mellitus. European journal of vascular and endovascular surgery. 2007;33(5):592-598. 
12- ANTONIOU G.A., GEORGIADIS G.S., ANTONIOU S.A., et al.: A meta-analysis of outcomes of endovascular abdominal aortic aneurysm repair in patients with hostile and friendly neck anatomy. J. Vasc. Surg., 57 (2): 527538,2013

13- MATSUMOTO T., TANAKA S., OKADOME J., et al.: Midterm outcomes of endovascular repair for abdominal aortic aneurysms with the on-label use compared with the off-label use of an endoprosthesis. Surgery Today, 45 (7): 880-885, 2015.

14- CERINI P., GUZZARDI G., DIVENUTO I., et al.: Are abdominal aortic aneurysms with hostile neck really unsuitable for EVAR? Our experience Radiol. Med., 121: 528-535, 2016.

\section{دور القسطرة العلاجية فى إصلاح التمدد الشريانى الأورطى الهمى



القسطرة العلاجية أصبحت خط العلاج الأول فى علاج مرضى التمدد الشريانى الأوطى ذو التشريح المناسب على عكس علاج مرضى التهى

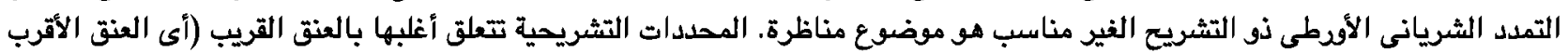

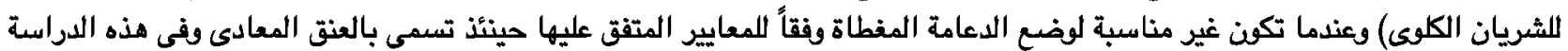

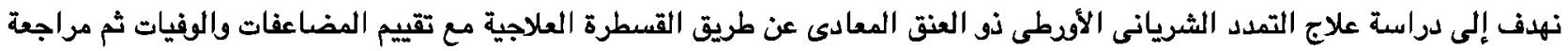

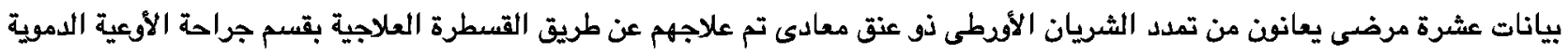

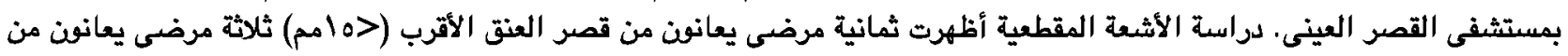

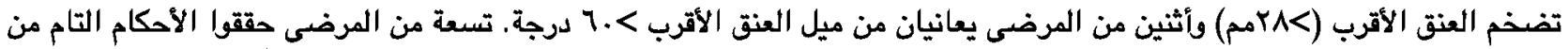

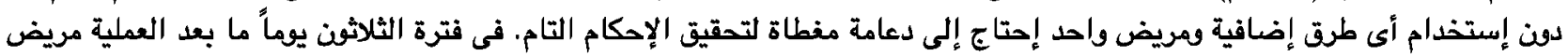

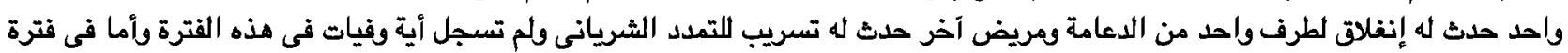

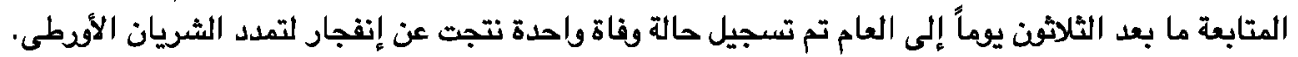

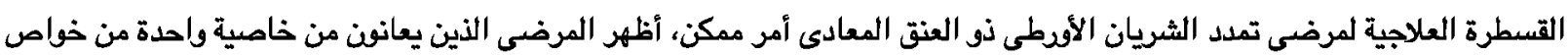

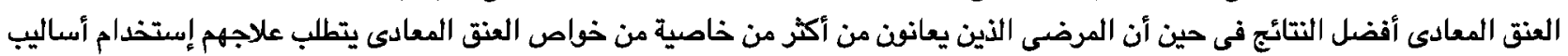

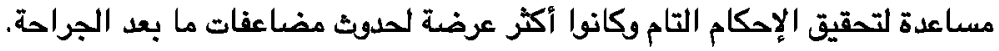

